\title{
Editorial
}

\section{Christian Colliex}

Laboratoire de Physique des Solides associé au CNRS, Bâtiment 510, Université Paris Sud, 91405 Orsay, France

For 14 years, the "Journal de Microscopie et de Spectroscopie Electroniques" has been the organ of the Société Française de Microscopie Electronique (S.F.M.E.), devoted to improvements in electron microscopy, diffraction and spectroscopy techniques and to their implementation in various fields of materials science. Even if we consider electron beam methods only, this period has been marked by spectacular evolution. Great changes have occurred, such as the merging into one analytical electron microscope of two activities with long parallel histories, electron microscopy and microanalysis. The burst of computer assistance has proved fruitful, through the development of image and spectral processing in all aspects of quantitative applications. Unexpected techniques have blossomed, the most famous being tunnelling microscopy and spectroscopy, which have dramatically modified our experimental approach to both perfect and real surfaces. These instrumental developments have resulted in a whole set of very high performance tools for the investigation of structural, chemical and electronic properties of matter with high spatial resolution. For instance, high resolution electron micrographs routinely provide atomic structure images of the real crystal, and microanalysis has stepped into the world of nanoanalysis.

Electron beams are one of many useful tools for the local study of materials. Field ion microscopy still remains the most powerful technique for single atom identification. Secondary ion emission microscopy is unique for isotopic discrimination. A wide range of scanning microscopies, with quite diverse probes (atomic force microscopy, near field optical microscopy, ultrasonic microscopy, ion conductance microscopy, capacitance microscopy...) have emerged for the investigation of many different properties of matter from the microscopic to the macroscopic domains.

Our theoretical understanding of the basic mechanisms involved in these different methodologies has likewise advanced, leading to improvements in the use and interpretation of data. The symbiosis between distinct fields of knowledge has often been advantageous : as an example, image processing techniques have benefited from tools developed in astronomy or geography.

In view of this situation, it follows that the content and presentation of the "Journal de Microscopie et de Spectroscopie Electroniques" must evolve in order to insure that scientific work in this area reaches its audience. Consequently, in early 1990, this Journal begins a new life and the following general rules have been decided :

1) The periodical remains the official publication of SFME and of the newly created "Cercle de Microanalyse". Its aim is to publish all original manuscripts dealing with developments in all aspects 
of microscopy and microanalysis and with their use in materials science (metals, semiconductors, inorganic materials, ceramics, catalysts, polymers, minerals...).

2) A new title has been chosen :

\section{MICROSCOPY, MICROANALYSIS, MICROSTRUCTURES}

Notwithstanding the fact that the title is in English, manuscripts written in English, French or German will be accepted, once they have been through the normal peer review system as in any international publication.

3) The journal will essentially welcome Regular Papers describing high quality original research work, both experimental and theoretical, in the above-mentioned fields. In order to satisfy the needs that have often been voiced, Review Papers by recognized experts on subjects of general interest and written in a didactic spirit, will be regularly included. Innovations will consist of the introduction of Short Notes for rapid publication, Letters from the Editors (or from readers) devoted to topical subjects and possibly a Manufacturer's Corner. For more information concerning these different possibilities, please consult the Instructions for Contributors.

4) The scientific policy of the journal will be decided by a Chief Editor, a member of the SFME, assisted by several Foreign Associate Editors to help in promoting the international coverage of the publication, and with an Editorial Board to supervise the quality of its scientific content.

5) Six issues are planned every year at regular two-monthly intervals, in order to secure rapid publication for all manuscripts and especially for Short Notes. Extra issues for Colloquia, either those regularly organized in the annual meetings of SFME or in others, may be added.

6) The publication and distribution of the Journal are made by the "Société Les Editions de Physique", which already plays this role for several other publications such as the Journal de Physique, the Revue de Physique Appliquée, Europhysics Letters, the Annales de Physique and the Journal d'Acoustique.

7) The scientific secretariat of the journal remains located at the office of the French Society of Electron Microscopy (S.F.M.E.), but manuscripts may be submitted either to that address or to any member of the Foreign Associate Editors Board.

Société Française de Microscopie Electronique

67, rue Maurice Günsbourg, 94205 Ivry sur Seine Cedex, France 